Gustavo Luiz Bastos Baptista

\title{
Gerenciamento de Mobilidade e Tratamento de Desconexão Baseado em SIP
}

Dissertação de Mestrado

Dissertação apresentada como requisito parcial para obtenção do título de Mestre pelo Programa de PósGraduação em Informática da PUC-Rio.

Orientador:

Prof. Markus Endler

Co-orientador: Prof. Vagner Sacramento

Rio de Janeiro, fevereiro de 2009 
Gustavo Luiz Bastos Baptista

\title{
Gerenciamento de Mobilidade e Tratamento de Desconexão Baseado em SIP
}

\begin{abstract}
Dissertação apresentada como requisito parcial para a obtenção do grau de Mestre pelo Programa de Pósgraduação em Informática do Departamento de Informática do Centro Técnico e Científico da PUCRio. Aprovada pela Comissão Examinadora abaixo assinada.
\end{abstract}

Prof. Markus Endler

Orientador

Departamento de Informática - PUC-Rio

Prof. Vagner José do Sacramento Rodrigues

Co-orientador Instituto de Informática - UFG

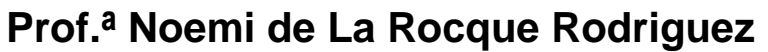

Departamento de Informática - PUC-Rio

Prof. Renato Fontoura de Gusmão Cerqueira

Departamento de Informática - PUC-Rio

Prof. José Eugenio Leal

Coordenador Setorial do Centro Técnico Científico - PUC-Rio 
Todos os direitos reservados. É proibida a reprodução total ou parcial do trabalho sem autorização da universidade, do autor e do orientador.

Gustavo Luiz Bastos Baptista

Bacharel em Informática pela Pontifícia Universidade Católica do Rio de Janeiro (PUC-Rio) em 2006. Atualmente, integra o grupo de pesquisadores do LAC (Laboratory of Advanced Collaboration) da PUC-Rio, desenvolvendo pesquisa na área de Sistemas Distribuídos.

Ficha Catalográfica

Baptista, Gustavo Luiz Bastos

Gerenciamento de mobilidade e tratamento de desconexão Baseado em SIP / Gustavo Luiz Bastos Baptista ; orientador: Markus Endler ; co-orientador: Vagner Sacramento. - 2009.

100 f. : il. ; $30 \mathrm{~cm}$

Dissertação (Mestrado em Informática)-Pontifícia Universidade Católica do Rio de Janeiro, Rio de Janeiro, 2009.

Inclui bibliografia

1. Informática - Teses. 2. Gerenciamento de mobilidade. 3. SIP. 4. Mobilidade de dispositivos. 5. Conectividade intermitente. 6. Middleware. I. Endler, Markus. II. Sacramento, Vagner. III. Pontifícia Universidade Católica do Rio de Janeiro. Departamento de Informática. IV. Título. 


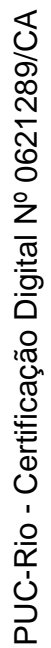

À minha mãe, Dilza e à minha amada esposa, Olympia. 


\section{Agradecimentos}

Ao Senhor Jesus Cristo. "O Senhor é a minha força e o meu escudo; nele o meu coração confia, nele fui socorrido; por isso, o meu coração exulta, e com o meu cântico o louvarei." Salmo 28:7

Ao Prof. Markus Endler, por todo o seu apoio, paciência, ensinamentos e oportunidades.

Ao Prof. Vagner Sacramento, por todos os ensinamentos, desde os tempos da minha graduação em que me recebeu no LAC, onde realizei meus estudos que resultaram neste trabalho.

À Prof ${ }^{\text {a }}$. Dilza Szwarcman, Prof ${ }^{\text {a }}$. Monica Costa e o Prof. Bruno Feijó, pela experiência e ensinamentos que adquiri trabalhando na Midiarte e no ICAD e pelo apoio para a realização deste mestrado.

À minha amada esposa Olympia, amor da minha vida, pela paciência, perseverança e apoio.

À toda a minha amada família. Meus pais, Dilza e Hilton Roberto, irmã, Daniela, avós Nice Baptista e Hilda Bastos, tias Ana Luiza Baptista e Solange Bastos e tio João Bastos. Em especial à minha mãe Dilza e à minha avó Nice, por todo o suporte em todas as áreas possíveis.

Em memória de meus avôs Hilton Baptista e Thomaz de Aquino Bastos meus grandes exemplos de vida, os quais eu gostaria que estivessem aqui para compartilhar esta conquista.

Ao meu primo Gerson Rocha, pela ajuda no momento em que mais precisei.

Aos queridos Ana Carla Rocha e Mozart Rocha, pelos bons momentos que passamos juntos e pelo apoio em momentos difíceis.

Ao Prof. Ricardo Rocha, por todos os seus ensinamentos e ajuda no LAC.

Aos demais amigos do LAC, Prof ${ }^{\text {a }}$. Hana Rubinsztejn, Giulliano Bueno, José Viterbo, Fernando Nascimento, Marcelo Malcher, Juliana Aquino e Jordan Janeiro, pelo tempo que passamos juntos, trocando experiências e bons momentos que ficarão para sempre na memória.

À PUC-Rio e ao Departamento de Informática, pelos auxílios financeiros concedidos, sem os quais este trabalho não poderia ter sido realizado. 


\section{Resumo}

Baptista, Gustavo Luiz B., Endler, Markus, Sacramento, Vagner. Gerenciamento de Mobilidade e Tratamento de Desconexão Baseado em SIP. Rio de Janeiro, 2009. 100 p. Dissertação de Mestrado Departamento de Informática, Pontifícia Universidade Católica do Rio de Janeiro.

Os avanços nas tecnologias de redes de computadores, telecomunicações, e dispositivos móveis portáteis têm gerado uma demanda por aplicações e serviços que sejam apropriados para ambientes com conectividade intermitente e mobilidade dos dispositivos. Uma questão central para a viabilização de tais aplicações em ambientes como estes são as possíveis soluções para o Gerenciamento de Mobilidade, isto é, a manutenção automática da conectividade entre componentes de um sistema em cenários nos quais os dispositivos mudam de endereço IP dinamicamente, à medida que se reconectam a diferentes domínios de rede. Partindo de uma investigação inicial das principais soluções existentes, este trabalho apresenta a implementação de uma solução na camada de aplicação baseada no protocolo SIP. Em seguida, apresenta a adaptação de um sistema publish/subscribe existente para fazer com que o mesmo utilize a solução implementada, com o objetivo de prover suporte à mobilidade e à desconexão dos produtores e consumidores de eventos, bem como para a travessia de firewalls e NATs, permitindo que o sistema publish/subscribe possa ser usado na Internet, e não somente em redes locais. $\mathrm{O}$ modelo publish/subscribe foi escolhido como estudo de caso, pois oferece interações assíncronas e anônimas que se adéquam muito bem aos requisitos de um cenário de mobilidade. A adaptação deste sistema partiu da investigação de alguns dos principais requisitos que um sistema pub/sub deve atender para tratar mobilidade e desconexão de dispositivos. O referido sistema foi testado para diferentes cenários, e seu desempenho foi avaliado para diferentes configurações, e comparado ao do sistema publish/subscribe convencional.

\section{Palavras-chave}

Gerenciamento de Mobilidade, SIP, publish/subscribe, Mobilidade de dispositivos, Conectividade Intermitente, Middleware 


\section{Abstract}

Baptista, Gustavo Luiz B., Endler, Markus, Sacramento, Vagner. Mobility Management and Disconnection Handling Based on SIP. Rio de Janeiro, 2009. 100 p. Master Thesis - Departamento de Informática, Pontifícia Universidade Católica do Rio de Janeiro.

Advances in computer networks, telecommunications, and portable mobile devices have increased the demand for applications and services that are suitable for environments with intermittent connectivity and mobility of devices. A central question for the viability of such applications on environments like these are the possible solutions for Mobility Management, i.e., the automatic maintenance of the connectivity between system components in scenarios where the devices change their IP addresses dynamically as they reconnect to different network domains. Starting from an initial investigation of the main solutions for Mobility Management, this dissertation presents the development of a solution on the application layer based on the SIP protocol. Then, it presents the adaptation of an existing publish/subscribe system to make it use the developed Mobility Management solution, in order to provide support for the mobility and disconnection of event producers and consumers, and also NAT and firewall traversal, enabling the system to be used on the Internet, and not only on local networks. The publish/subscribe model has been chosen as a case study for the implemented solution, because it provides asynchronous and anonymous interactions that are very well suited to the requirements of a mobility scenario. The adaptation of the publish/subscribe system started from an investigation of the main requirements a system like this should meet in order to support mobility and disconnection of devices. The referred system has been tested for different scenarios, and its performance has been evaluated for different configurations, and it has been compared to the conventional publish/subscribe system.

\section{Keywords}

Mobility Management, SIP, publish/subscribe, Terminal Mobility, Intermittent Connectivity, Middleware 


\section{Sumário}

1 Introdução 13

$\begin{array}{lr}\text { 1.1. Objetivo } & 17\end{array}$

1.2. Contribuições 18

2 Fundamentação Conceitual 19

2.1. Session Initiation Protocol (SIP) 19

2.2. Utilização do Protocolo SIP através de redes com NAT (Network Address Translation) 25

2.3. Sistemas Publish/Subscribe 33

3 Gerenciamento de Mobilidade 38

3.1. Suporte Abaixo da Camada de Rede $\quad 40$

3.2. Gerenciamento de Mobilidade na Camada de Rede 41

3.3. Gerenciamento de Mobilidade Entre as Camadas de Rede e de Transporte $\quad 42$

3.4. Gerenciamento de Mobilidade na Camada de Transporte 44

3.5. Gerenciamento de Mobilidade na Camada de Aplicação 47

4 Desenvolvimento de uma Solução de Gerenciamento de Mobilidade na Camada de Aplicação com SIP 50

5 Gerenciamento de Mobilidade Aplicado a um Sistema Publish/Subscribe

5.1. Requisitos de um Sistema Publish/Subscribe para Suportar Mobilidade e Desconexão

5.2. Implementação dos Requisitos do Sistema

Publish/Subscribe para Suportar Mobilidade e Desconexão 
6 Avaliação e Testes 79

6.1. Testes de Funcionamento da API SIP User Agent 79

6.2. Testes de Funcionamento do Sistema Pub/Sub com

Gerenciamento de Mobilidade $\quad 80$

6.3. Testes de Desempenho 82

7 Trabalhos Relacionados $\quad 87$

7.1. Utilização de SIP para Gerenciamento de Mobilidade 87

7.2. Mobilidade em Sistemas Publish/Subscribe 88

8 Conclusão e Trabalhos Futuros 92

9 Referências Bibliográficas 95 


\section{Lista de figuras}

Figura 1 - Exemplo clássico de estabelecimento de sessão

"Trapezóide SIP" (Rosenberg, Schulzrinne et al., 2002) 23

Figura 2 - Exemplo de Requisição INVITE com descrição SDP 24

Figura 3 - Mecanismo de NAT entre uma rede privada e uma rede pública (Cisco, 2006)

Figura 4 - Exemplo de NAT estático mapeando endereços IP privados para endereços IP públicos de forma estática e um para um (Cisco, 2006)

Figura 5 - Exemplo de NAT dinâmico utilizando um pool de endereços (Cisco, 2006)

Figura 6 - Exemplo de NAT simétrico

Figura 7 - Exemplo de mensagem SIP enviada a partir de um User Agent executando em um dispositivo anexado a uma sub-rede com NAT.

Figura 8 - Exemplo de cenário com utilização de um

Media Proxy para travessia de NAT

Figura 9 - Exemplo de descrição SDP de uma requisição

SIP recebida pelo SIP Proxy, em que os endereços IP e portas contidos na descrição SDP são substituídos

Figura 10 - Modelo de comunicação publish/subscribe

(Eugster, Felber et al., 2003)

Figura 11 - Arquitetura centralizada vs. arquitetura

distribuída para sistemas publish/subscribe

Figura 12 - Funcionamento da solução de Gerenciamento de Mobilidade na camada de rede Mobile IP

Figura 13 - Camada HIP entre as camadas de rede e de transporte (Bondareva e Baumann, 2006)

Figura 14 - Estabelecimento de uma associação HIP 
(Bondareva e Baumann, 2006)

Figura 15 - Exemplo de associação SCTP com um caminho

primário e um caminho secundário (Fu, Atiquzzaman et al., 2004)

Figura 16 - Exemplo de mobilidade com o protocolo SIP

(Wedlund e Schulzrinne, 1999)

Figura 17 - Módulos da API SIP User Agent $\quad 50$

Figura 18 - Principais métodos da fachada SIPUserAgent 51

Figura 19 - Principais métodos da interface SIPUAListener 52

Figura 20 - Principais componentes da API JAIN SIP

(O'doherty e Ranganathan, 2003)

Figura 21 - Exemplo de dados de comunicação SIP

(SIP Communication Data) e dados de mídia (Media Data)

de uma Sessão SIP

Figura 22 - Estados de uma Sessão SIP durante o estabelecimento da mesma.

Figura 23 - Exemplo de Sessão de Comunicação de um usuário.

Figura 24 - Estabelecimento dos componentes do

MD-ECl em um ambiente de mobilidade.

Figura 25 - Utilização da API SIP User Agent pelo ECIAgent e ECIBroker

Figura 26 - Registro no Servidor SIP da rede doméstica do usuário

Figura 27 - Estabelecimento de uma Sessão entre o

ECIAgent e o ECIBroker

Figura 28 - Gerenciamento de Sessões de Comunicação

Figura 29 - Exemplo de Subscrição identificada pelo

URI do usuário a quem ela pertence

Figura 30 - Recuperação de uma Sessão após a

mobilidade de um publicador/subscritor

Figura 31 - Detecção da desconexão pelo ECIAgent e ECIBroker $\quad 74$

Figura 32 - Subscrição com Tempo de Expiração especificado

Figura 33 - Subscrição com uma Restrição de Geração de Notificações Temporal de Freqüência limitando a freqüência de geração de Notificações para 5 minutos. 
Figura 34 - Subscrição com uma Restrição de Geração de Notificações Temporal de Intervalo de Tempo limitando a geração de Notificações para um determinado horário diário.

Figura 35 - Interface gráfica com o usuário (GUI) da aplicação de testes da API SIP User Agent

Figura 36 - Aplicação de testes da API ECIAgent com suas principais funcionalidades expostas para o usuário Figura 37 - Tela auxiliar da aplicação de testes da API ECIAgent, onde as notificações recebidas podem ser visualizadas

Figura 38 - Tela auxiliar da aplicação de testes da API ECIAgent, onde informações sobre a utilização da API SIP User Agent e a sessão de comunicação com o ECIBroker podem ser visualizadas

Figura 39 - Avaliação do overhead causado pelo

Gerenciamento de Mobilidade.

Figura 40 - Avaliação do desempenho do MD-EDI no Android/G1

86

Figura 41 - Mobilidade em sistemas publish/subscribe com arquitetura distribuída. 